\title{
Simultaneous measurement of position and color of single fluorescent emitters using diffractive optics
}

\author{
Jordi Broeken, Bernd Rieger, and Sjoerd Stallinga* \\ Department of Imaging Physics, Delft University of Technology, 2628 CJ Delft, The Netherlands \\ ${ }^{*}$ Corresponding author s.stallinga@tudelft.nl
}

Received December 20, 2013; revised April 15, 2014; accepted April 27, 2014;

posted May 2, 2014 (Doc. ID 203544); published May 30, 2014

\begin{abstract}
We propose a method for simultaneously measuring the position and emission color of single fluorescent emitters based on the use of a large pitch diffraction grating in the emission light path. The grating produces satellite spots adjacent to the main spot; the relative distance between the spots is a measure for the emission wavelength. We present proof-of-principle experiments on beads and mixtures of quantum dots using a spatial light modulator for making a programmable diffraction grating. A wavelength precision of around $10 \mathrm{~nm}$ can be achieved for 1000 signal photons and practical background levels, while maintaining a localization precision of around $10 \mathrm{~nm}$. () 2014 Optical Society of America

OCIS codes: (180.2520) Fluorescence microscopy; (100.6640) Superresolution; (050.1950) Diffraction gratings; (230.6120) Spatial light modulators; (300.0300) Spectroscopy.

http://dx.doi.org/10.1364/OL.39.003352
\end{abstract}

Super-resolution microscopy based on the localization of stochastically activated single fluorescent molecules is a powerful technique for studying biological structure on the nanoscale [1-3]. The study of biological function on this scale requires imaging two or more interacting molecular species. These species are usually differentiated by labeling with fluorescent molecules that have different emission colors. The different species can be imaged subsequently by changing illumination and filter sets []ㅡ or simultaneously by using multibandpass filters and a dichroic beamsplitter in the emission path for imaging two color channels simultaneously $[5,6]$. The latter approach allows for multiplexing three and, in some cases, four different fluorescent species. An alternative is the use of activator-reporter labeling techniques in which different activator molecules can couple to the same reporter molecule, thereby allowing for subsequent imaging of the different channels by switching the illumination wavelength only [7]. Multicolor localization approaches are also applied in single particle tracking, broadening the scope to emitters such as quantum dots (QDs) [8].

A different approach to color measurement is enabled by placing a blazed diffraction grating with a fine pitch in the emission path [ㅁ, 10]. In this way two subimages, corresponding to the zeroth and first diffraction orders, are imaged side by side on two camera halves. The 0th order image gives the positions of the emitters; the 1st order image is smeared out in proportion to the emission spectral width over many pixels, thus enabling spectroscopic measurements. The resulting low signal-to-background ratio (SBR), however, compromises spectral and localization precision. Moreover, differentiation between species does not necessarily require measuring the full emission spectrum. Here, we propose a new method for measuring both the position and the color of single emitters based on the use of a grating with a relatively large pitch. The grating produces a set of satellite diffraction orders directly adjacent to the 0th order, the main spot. Now the satellite order(s) are projected just a handful of pixels away instead of to the other half of the camera. A consequence is that spot broadening due to the emission spectral width is at the subpixel level. The distance between the diffraction spots $\Delta x=\lambda z / p$, with $p$ the pitch and $z$ the propagation distance, is now a direct measure for the peak emission wavelength $\lambda$ and can be used to differentiate molecular species.

Generally, the diffraction grating splits the converging beam in a set of diffraction orders with integer index $j$ and with diffraction efficiency $\eta_{j}$, producing a set of subspots on the detector at coordinates $x_{j}=x_{\mathrm{em}}+j z \lambda / p$ and $y_{j}=y_{\mathrm{em}}$. The light distribution on the detector is modeled as a sum of mutually displaced Gaussians:

$$
I(x, y)=\sum_{j} \frac{N_{j}}{2 \pi \sigma^{2}} e^{-\frac{\left(x-x_{j}\right)^{2}+\left(y-y_{j}\right)^{2}}{2 \sigma^{2}}}+\frac{b}{a^{2}} .
$$

Here, $b$ is the number of background photons per pixel, $a^{2}$ the pixel area, $\sigma$ the spot width, and $N_{j}=\eta_{j} N$ is the signal photon count of diffraction order $j$, with $N$ the total signal photon count. The precision for finding the center positions of the different orders is $\Delta x_{j}=$ $\sigma_{a} / \sqrt{N_{j}\left(1-I_{1}\left(\tau_{j}\right)\right)}$, and the precision for finding the photon counts for each diffraction order is $\Delta N_{j}=\sqrt{N_{j} /\left(1-I_{0}\left(\tau_{j}\right)\right)}$, with the functions $I_{n}(\tau)=$ $(\tau / n !) \int_{0}^{\infty} \mathrm{d} t t^{n} /\left(1+\tau e^{t}\right)$ of the dimensionless background parameter $\tau_{j}=2 \pi \sigma_{a}^{2} b / N_{j} a^{2}$, where $\sigma_{a}^{2}=\sigma^{2}+a^{2} / 12$ $[\underline{11}, \underline{12}]$. A Cramér-Rao lower bound (CRLB) analysis gives the best possible estimate for the emitter position and the wavelength as:

$$
\begin{gathered}
\frac{1}{\left(\Delta x_{\mathrm{em}}\right)^{2}}=\sum_{j}\left(\frac{\partial x_{j}}{\partial x_{\mathrm{em}}}\right)^{2} \frac{1}{\left(\Delta x_{j}\right)^{2}}=\sum_{j} \frac{N_{j}}{\sigma^{2}}\left(1-I_{1}\left(\tau_{j}\right)\right), \\
\frac{1}{(\Delta \lambda)^{2}}=\sum_{j}\left[\left(\frac{\partial x_{j}}{\partial \lambda}\right)^{2} \frac{1}{\left(\Delta x_{j}\right)^{2}}+\left(\frac{\partial N_{j}}{\partial \lambda}\right)^{2} \frac{1}{\left(\Delta N_{j}\right)^{2}}\right] \\
=\sum_{j} N_{j}\left[\left(\frac{j z \sigma}{p}\right)^{2}\left(1-I_{1}\left(\tau_{j}\right)\right)+\left(\frac{1}{\eta_{j}} \frac{\partial \eta_{j}}{\partial \lambda}\right)^{2}\left(1-I_{0}\left(\tau_{j}\right)\right)\right] .
\end{gathered}
$$


The $y$-position of the emitter can be estimated with the same precision as the $x$-position, even though the light distribution itself is anisotropic. The increase in spot footprint is comparable to the different methods for 3D-localization [12].

These predictions are tested with simulations, using realistically simulated spot shapes including effects of vector diffraction [13] and of a finite spectral emission bandwidth (Gaussian shape, FWHM = $35 \mathrm{~nm}$ ). Modeled spots with added shot noise were fitted with a maximum likelihood estimator using the model of Eq. (1) and a Levenberg-Marquardt optimizer. Assumed parameters were a nominal wavelength $\lambda=500 \mathrm{~nm}$, a numerical aperture $\mathrm{NA}=1.25$, and a pixel size in object space $a=80 \mathrm{~nm}$. In the simulations we have assumed the use of a binary phase grating with $50 \%$ duty cycle giving efficiencies $\eta_{0}=$ $\cos ^{2}(\pi \Delta L / \lambda)$ and $\eta_{ \pm 1}=\left(4 / \pi^{2}\right) \sin ^{2}(\pi \Delta L / \lambda)$ with $\Delta L=$ $150 \mathrm{~nm}$ the path length difference between the two levels. Only the central 3 diffraction orders are taken into account into the fitting. Outliers were removed from the statistical evaluation based on fitted spot width (more than twice the pixel size), fitted position (more than 2 pixels away from the center of the fit region), and final merit function value (less than 3 standard deviations below the average). Figure 1 shows the precision of the position and wavelength estimation as a function of signal photon count for different levels of background. The standard deviation over the full set of 500 randomized instances agrees with the CRLB within statistical error except when the SBR is too low. In addition, a sizeable portion of the fits is designated as outliers in this regime of very low SBR. For zero background the localization uncertainty is the same as for conventional single spot Gaussian fitting; for nonzero background levels the localization uncertainty is compromised due to more unfavorable local SBR for the three subspots. Good results can be obtained for signal photon counts above 200-500 photons and background levels below 10 photons per pixel. There is a small bias of a few nanometer in the determination of the wavelength due to model errors; the wavelength precision is on the order of $5-10 \mathrm{~nm}$ for the signal and background photon levels quoted above.

Proof of principle experiments have been done with the setup shown in Fig. 2. A liquid crystal on silicon (LCOS) based spatial light modulator (SLM, Holoeye LC-R 720, pixel size $20 \mu \mathrm{m}$ ) is placed in the Fourier plane of a 4f-relay by using two achromats (Thorlabs AC508200-A) on the exit port of a Nikon N-STORM microscope equipped with a $100 \times /$ NA1.45 APO-TIRF objective and
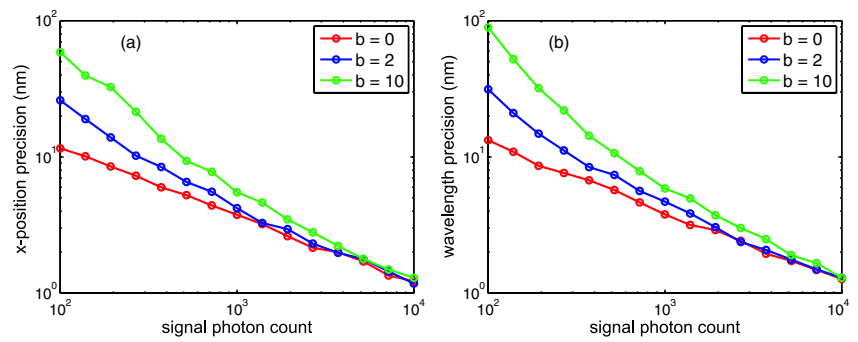

Fig. 1. Simulation results of the emitter position (a) and wavelength (b) precision as a function of signal photon count for three different background levels.

$1.5 \times$ tube lens. The SLM is set to produce a binary phase grating with path length difference $\Delta L$ and pitch $p$ for one polarization state, whereas the other polarization state is unaffected. This results in diffraction efficiencies $\eta_{0}=$ $\left(1+\cos ^{2}(\pi \Delta L / \lambda)\right) / 2$ and $\eta_{ \pm 1}=\left(2 / \pi^{2}\right) \sin ^{2}(\pi \Delta L / \lambda)$. Images were recorded on an Andor iXon3 897 EMCCD camera (pixel size $16 \mu \mathrm{m}$ ), converted to photoelectron counts via a gain calibration and processed using an adaptation of the method of ref. [14] for finding candidate regions, and analyzed with the MLE-fitting routine.

Time lapse measurements (250 frames of each $60 \mathrm{~ms}$ exposure time) of a set of 83 beads (ThermoScientific $50 \mathrm{~nm}$ diameter polystyrene beads, peak emission wavelength $612 \mathrm{~nm}$ ) are recorded with an SLM with pitch set to 60 pixels $(p=1.2 \mathrm{~mm})$. Figure $2(\mathrm{~b})$ shows the average of the measured spots over all $83 \times 250=20750$ events, after registration and upsampling with a factor of 5 (for display purposes), Fig. 2(c) shows the average of the fitted Gaussian spots, Fig. 2(d) shows cross-sections of the average measured and fitted spots. The Gaussian spot model fits the observations rather well, although a non-Gaussian spot shoulder is clearly visible. This may be attributed to vector diffraction effects and to stray light originating from the SLM. In addition, the average measured spot is not rotationally symmetric (a Gaussian fit on the average measured spot gives spot widths $\sigma_{x}=$ $164 \mathrm{~nm}$ and $\sigma_{y}=141 \mathrm{~nm}$ ), indicating the presence of astigmatism. The path length difference $(\Delta L=0.3 \mu \mathrm{m}$, corresponding to a phase depth $\pi$ ) is obtained from the relative peak heights of the measured average diffraction

(a)
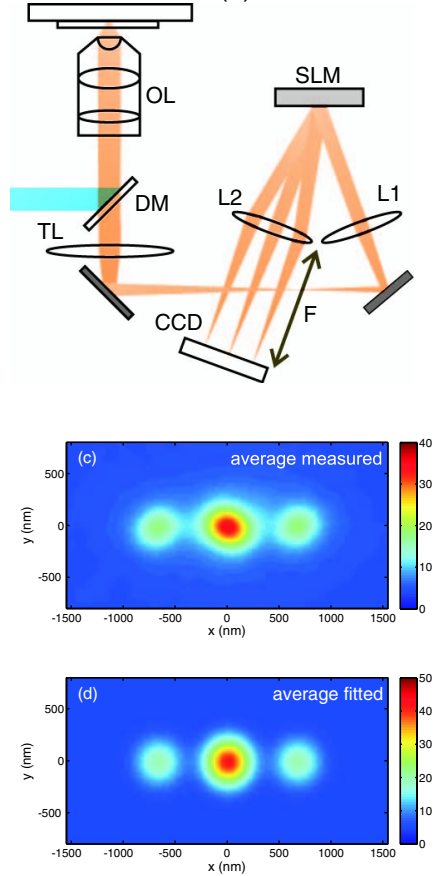

Fig. 2. (a) Schematic overview of experimental setup. The sample is illuminated via a dichroic mirror (DM) and objective lens (OL), fluorescent light is captured and imaged onto the CCD via the tube lens (TL) and a 4f-relay of achromats L1 and L2 with the SLM in the pupil plane. (b) Example image of set of beads. (c) Average measured spot, after registration and upsampling, (d) average fitted spot, and (e) cross-sections of the average measured spot and average fitted spot. 
spots. The set of fits for the individual frames is grouped in 10 sets of 25 consecutive fits. The standard deviation of the fitted parameters over the 25 consecutive fits and the mean of the CRLB-based precision, evaluated for the fit parameters at the found optimum values are subsequently calculated. These values are binned in 10 bins (following Scott's normal reference rule [15]) with mean signal photon count in the range 300-3000, equidistant on a logarithmic scale. The mean and standard deviation of the error and the CRLB precision within these bins are plotted in Fig. 3. The localization precision in the $y$-direction was typically a few percent worse than the localization precision in the $x$-direction, probably due to the astigmatism. The found error is slightly above the CRLB, implying close to optimum fitting conditions. The deviations may be caused by the model errors described above. The found fit precision also agrees quite
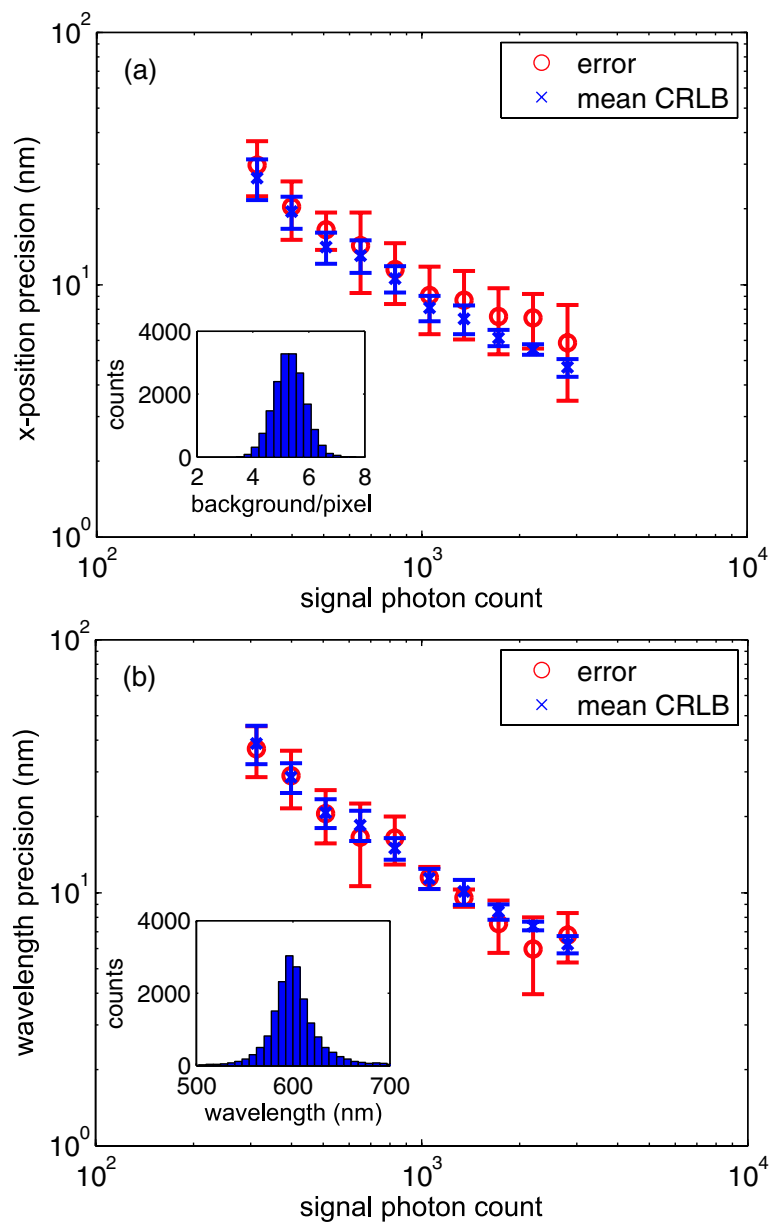

Fig. 3. Experimental results. (a) Precision of the $x$-position as a function of signal photon count showing agreement between the localization error determined from 25 consecutive fits with the mean precision according to the CRLB evaluated for the fitted parameter values. The inset shows a narrow distribution of background photon counts with an average of 5-6 photons per pixel. (b) Precision of the wavelength as a function of signal photon count showing agreement between the error determined from 25 consecutive fits with the mean precision according to the CRLB evaluated for the fitted parameter values. The inset shows the histogram over all fitted wavelength values for the individual fits, with a peak value around $590 \mathrm{~nm}$, giving a small bias with the expected value of $612 \mathrm{~nm}$. well with the simulation results, even though the dependence of the diffraction efficiency on $\lambda$ is different. The reason is that the diffraction efficiencies carry little information on the wavelength compared to the satellite spot distance, especially for path length differences close to $\lambda / 2$. The histogram of fitted wavelengths (peak around $590 \mathrm{~nm}$ ) indicates a bias in the results compared to the manufacturer specification (peak emission at $612 \mathrm{~nm}$ ). This bias can be attributed to model errors (also found in the simulations), to an error within the specification tolerance of the focal length of the relay achromats, or to relay branch misalignment.

Next, mixtures of different QDs (Invitrogen Streptavidin Conjugate, with peak emission wavelengths at 525, $565,585,605,625,655,705$, and $800 \mathrm{~nm}$ ) were imaged. Figure 4(a) shows an image taken with our setup, and Fig. 4(b) shows a reference image taken with a consumer color photo camera (Nikon D40) attached to the other side port of the microscope. A mixture of three relatively bright QDs (QD605, QD655, and QD705) was then made for analysis with the MLE-fitting routine. Figure 4(c) shows a histogram of found wavelengths and Gaussian fits of the three peaks. Clearly, a small fraction of the QDs present was found to be QD655. The widths of the peaks for QD605 (12 $\mathrm{nm})$ and QD655 $(10 \mathrm{~nm})$ are similar to the mean wavelength error $(16 \mathrm{~nm}$, determined from repeated fits on the same QDs in consecutive frames), the peak width for QD705 (33 nm) is quite a bit larger. Sample heterogeneity is not needed to explain the observed peak widths for QD605 and QD655; it possibly is for the peak for QD705. Events are classified as belonging to one of the three $\mathrm{QD}$ species based on the highest a priori likelihood given the estimated wavelength. This classification is thus defined by the intersections of the three normalized Gaussian probability distribution functions of the three QD species. The resulting error rates for the three species of QDs are then 5.6\%, 22\%, and 8.9\%.
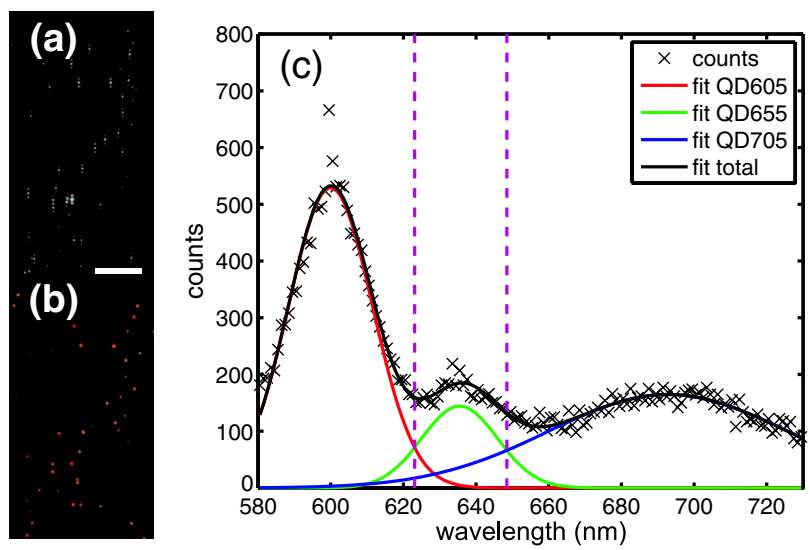

Fig. 4. (a) Image of a mixture of 8 different species of QDs (scalebar $10 \mu \mathrm{m}$ ). (b) Reference image of same area taken with a consumer color camera attached to the other side port of the microscope. Differences between (a) and (b) are due to differences in spectral sensitivity and blinking. (c) Histogram of found wavelengths from a time lapse measurements of a mixture of three species of QDs (QD605, QD655, QD705), with Gaussian fits of the three peaks [with widths of $600(12) \mathrm{nm}$, $635(10) \mathrm{nm}$, and 693(33) nm]. The magenta vertical lines at $623 \mathrm{~nm}$ and $648 \mathrm{~nm}$ indicate lines for classifying the three color species based on the estimated wavelength. 
More sophisticated classifiers developed for ratiometric detection of emission color $[\underline{16}, \underline{17}]$ may be tried as well in this context.

In conclusion, we have presented a method for simultaneously measuring the position and emission wavelength of single emitters using diffractive optics. Next steps are improving the technique by reducing aberrations and stray light originating from the SLM, optimizing the grating shape (e.g., using a blazed or sinusoidal shape), and providing an extension to 3D-localization. The practical value of the technique would be greatly enhanced by using a fixed glass-etched grating that can be slid in and out of the optical path, instead of the current SLM in a relay branch. Such a simplified setup would solve the aforementioned performance issues with the SLM and would open the route for differentiating more than four fluorescent species.

Keith Lidke is acknowledged for assistence with quantum dot samples. This research is supported by the Dutch Technology Foundation (STW), which is part of the Netherlands Organisation for Scientific Research (NWO) and which is partly funded by the Ministry of Economic Affairs, Agriculture and Innovation.

\section{References}

1. E. Betzig, G. H. Patterson, R. Sougrat, O. W. Lindwasser, S. Olenych, J. S. Bonifacino, M. W. Davidson, J. LippincottSchwartz, and H. F. Hess, Science 313, 1642 (2006).

2. M. J. Rust, M. Bates, and X. Zhuang, Nat. Methods 3, 793 (2006).
3. S. T. Hess, T. P. Girirajan, and M. D. Mason, Biophys. J. 91, 4258 (2006).

4. H. Shroff, C. G. Galbraith, J. A. Galbraith, H. White, J. Gillette, S. Olenych, M. W. Davidson, and E. Betzig, Proc. Natl. Acad. Sci. USA 104, 20308 (2007).

5. M. Bossi, J. Folling, V. N. Belov, V. P. Boyarskiy, R. Medda, A. Egner, C. Eggeling, A. Schonle, and S. W. Hell, Nano Lett. 8, 2463 (2008).

6. I. Testa, C. A. Wurm, R. Medda, E. Rothermel, C. von Middendorf, J. Fölling, S. Jakobs, A. Schönle, S. W. Hell, and C. Eggeling, Biophys. J. 99, 2686 (2010).

7. M. Bates, B. Huang, G. T. Dempsey, and X. Zhuang, Science 317, 1749 (2007).

8. P. J. Cutler, M. D. Malik, S. Liu, J. M. Byars, D. S. Lidke, and K. A. Lidke, PLoS One 8, e64320, 2013.

9. Y. Ma, M. R. Shortreed, and E. S. Yeung, Anal. Chem. 72, 4640 (2000).

10. G. A. Blab, S. Oelllerich, R. Schumm, and T. Schmidt, Opt. Lett. 29, 727 (2004).

11. K. I. Mortensen, L. S. Churchman, J. A. Spudich, and H. Flyvbjerg, Nat. Methods 7, 377 (2010).

12. B. Rieger and S. Stallinga, Chem. Phys. Chem. 15, 664 (2014).

13. S. Stallinga and B. Rieger, Opt. Express 18, 24461 (2010).

14. A. Sergé, N. Bertaux, H. Rigneault, and D. Marguet, Nat. Methods 5, 687 (2008).

15. D. W. Scott, Biometrika 66, 605 (1979).

16. M. S. Gunewardene, F. V. Subach, T. J. Gould, G. P. Penoncello, M. V. Gudheti, V. V. Verkhusha, and S. T. Hess, Biophys. J. 101, 1522 (2011).

17. D. Baddeley, D. Crossman, S. Rossberger, J. E. Cheyne, J. M. Montgomery, I. D. Jayasinghe, C. Cremer, M. B. Cannell, and C. Soeller, PloS One 6, e20645 (2011). 